\title{
LA REPRÉSENTATION DU DISCOURS DE GUSTAVE FLAUBERT DANS UNE ÉTUDE DE FERDINAND BRUNETIĖRE
}

\begin{abstract}
A bstract. Dutka Anna, La représentation du discours de Gustave Flaubert dans une étude de Ferdinand Brunetière [Gustav Flaubert's discourse in Ferdinand Brunetière's study]. Studia Romanica Posnaniensia, Adam Mickiewicz University Press, Poznań, vol. XXV/XXVI: 2000, pp. 81-88. ISBN 83-232-0965-0, ISSN 0137-2475.
\end{abstract}

The analysis concerns the means of representation of the discourse of G. Flaubert in the study of F. Brunetière which has as its aim the assessment of this writer on the basis of his works. The categories which have been considered are: representation of the other discourse (reported speech, autonymic modalisation) and the borrowing of segments which serve to discuss the procedures of writing. We have observed the presence of many opaque and clouding formulations. To a large degree the critic bases his judgements on the words and opinions of the writer himself. In particular cases, Flaubert as a source becomes cospeaker and is identified as «the poet».

Dans le cadre des cours consacrés à la littérature française, on présente aux étudiants les grands courants de la critique et la discussion des idées des critiques majeurs, on s'appuie sur les textes choisis (cf. Kroker, Sobieska-Piłatowicz 1997).

Pour les étudiants qui pendant des cours de linguistique s'intéressent à des analyses des textes concrets, nous proposons un exercice qui mettrait en évidence la dimension proprement linguistique et discursive des articles critiques. De quelle manière tel critique convoque-t-il les discours extérieurs au sien, et en particulier l'oeuvre de l'écrivain dont il parle?

Nous avons choisi une étude de Ferdinand Brunetière sur G. Flaubert, publiée dans le volume Le naturalisme français.

C'est la surface textuelle qui nous intéresse, et notre recherche s'appuie sur la notion d'hétérogénéité montrée: «Dans le fil du discours que produit, de fait, matériellement, un locuteur unique, un certain nombre de formes linguistiquement appréhendables au niveau de la phrase ou du discours inscrivent, dans la linéarité, de l'autre» (Authier-Revuz 1982: 91-92). Ici, «l'autre» est à entendre comme «le dire de Flaubert». 
Nous distinguons d'une part, entre REPRÉSENTER un discours autre et y RÉFÉRER (p.ex. Mortara-Garavelli 1985: 41 sv), et d'autre part, entre présenter des énoncés comme susceptibles de correspondre des ACTES D'ÉNONCIATION - ou bien, les présenter p.ex. comme des segments EMPRUNTÉS AUX TEXTES, p.ex. pour donner des exemples des procédés littéraires. Dans cette communication, nous envisageons les moyens linguistiques et discursifs utilisés par Brunetière par lesquels il renvoie au discours de Flaubert, leur emploi étant subordonné à l'objectif du critique: juger l'écrivain «sur ses oeuvres, sans esprit d'inutile flatterie, comme sans intention de vain dénigrément».

C'est la problématique du discours rapporté qui se trouve au coeur de notre analyse. Rappelons la réorientation des recherches dans ce domaine: la distinction traditionnelle en discours direct, discours indirect (formes grammaticales) et discours indirect libre (forme stylistique) se trouve modifiée grâce à l'adjonction du discours direct libre (sans guillemets et sans contexte introducteur). Le système des types de rapport se trouve rééquilibré et enrichi (Gaulmyn 1983, Simonin 1984, De Arruda 1992, Mortara-Garavelli 1985, 1995, Maingueneau 1994, Perrin 1994, Rosier 1997, Jaubert 1997), ce qui va de pair avec un élargissement des critères: on envisage les facteurs pragmatiques et rhétoriques (types de textes) au même titre que les éléments déictiques. On est amené à voir le DR comme un phénomène «langagier plutôt que linguistique» (De Arruda 1992: 191).

En refusant d'opposer le DD-citation au DI-reformulation par le rapporteur, les chercheurs optent pour une mixité de ces types, ce qui met en question la distinction basée sur la fidélité du rapport et l'idée de l'irréductibilité de ces formes.

Dans notre analyse nous envisageons:

\section{- La représentation du discours autre:}

1. Les formes que prend dans notre article divers types du discours rapporté: le discours direct (DD), le discours indirect (DI), le discours indirect libre (DIL), le discours narrativisé (DN) et la modalisation en discours second sur le contenu (MDSc) (terme de Authier-Revuz 1992), dit aussi discours indirect non subordonné (terme de Mortara-Garavelli 1995:72); il s'agit des syntagmes comme «d'après, pour, selon...+ nom/pronom».

2. La modalisation autonymique en discours second (MA): la modalisation en discours second sur les mots. C'est une configuration énonciative de l'auto-représentation du dire, qui dans un sous-ensemble de ses formes est susceptible de renvoyer aux mots du discours autre (la non-coinncidence du discours à lui-mễme): a) explicitement (des marques segmentales, comme «comme dit X, pour parler comme Z", etc.), b) intérprétativement (signaux typographiques, guillemets, italique) (Authier-Revuz, 1997: 36). C'est seulement ce type de MA qui est adéquat à notre analyse.

- Les emprunts des segments (terme de Moirand 1988: 320): nous entendons par là l'introduction des citations plus ou moins longues qui servent à discuter les procédés de l'écrivain. 
D'une manière générale, Brunetière indique Flaubert comme source de l'énonciation rapportée soit par le nom, soit par le pronom anaphorique «il», soit encore en citant ses personnages. Une fois (15)* il en fait son co-énonciateur en employant le pronom «vous».

Nous rappellerons brièvement les propriétés des types de rapport avant de commenter nos exemples. Nous indiquerons leur fonctionnement argumentatif.

Le discours direct (DD) est syntaxiquement autonome, les déictiques qu'il contient s'intérprètent par rapport à l'énonciation citée; le locuteur fait mention de celle-ci et par là il feint l'objectivité. Le DD est délimité du syntagme introducteur, mais la voix du rapporteur peut se manifester à tous les niveaux, aussi dans le DD lui-même.

Le DD dans notre corpus est signalé par une formule insérante antéposée $(1,4$, 7) placée en incise $(2,3,5)$ ou postposée (6). L'identité de Flaubert en tant qu'énonciateur rapporté est soulignée par l'adjonction de «-même» (dit-il lui-même (2), c'est lui-même qui l'a dit, et très bien dit (6), il l'a dit lui-même (7)). Les citations ainsi encadrées sont reprises par le critique au niveau argumentatif, en tant que source des principes pour juger Flaubert et ses oeuvres. Ainsi le critique reprend l'éloge pour la qualité de la description (2), l'idée que le mépris pour les gens empêche de créer (6), et que l'aspect noble de la réalité est peu spectaculaire (7). Dans un cas (4) on a un DD forgé par Brunetière, attribué à l'écrivain (C'est à peu près comme s'il avait dit:) et présenté comme une reformulation qui enchaîne sur une énonciation de Flaubert (8). La reprise présente un point de vue intenable et permet de conclure à «une lacune dans la connaissance de l'homme» chez Flaubert, liée selon Brunetière aux principes du naturalisme qu'il combattait.

Les enchaînements qui suivent le DD se laissent regrouper de la manière suivante: a) la particule «oui» et un point d'exclamation dans le segment qui suit $(1,7)$. Ce segment reprend les mots cités et développe la citation; b) une anaphore conceptuelle (2: «le mérite qu'il signale», 5: «sa devise»); c) une question rhétorique qui met en question le bien-fondé d'une affirmation de Flaubert (3); d) une conclusion explicite où l'adverbe «certainement» marque une déduction (4).

L'enchaînement sur la citation dans (1) peut être vu comme une restitution en DIL des pensées de l'héroïne, pour mieux faire ressortir la valeur poétique de l'imparfait, dans l'extrait où Emma Bovary se rend compte qu'elle s'était trompée dans le choix du mari. Progressivement, le critique passe à une formulation plus abstraite ( «flottant elle-même, pour ainsi dire, entre le regret des bonheurs qui ne reviendront plus et le charme si profondément humain de s'en souvenir!», p.161) qui lui permet de parler d' «une veine de poésie» (dans cet article, Flaubert a aussi le statut de poète, (18)). Nous avons ici une suite DD-DIL-énoncé primaire comme un moyen de passer de l'oeuvre au discours propre du critique.

* Remarque: les chiffres entre parenthèses renvoient aux exemples, à la fin de l'article, les pages localisent telle expression dans le texte de Brunetière. 
Le discours indirect (DI) comporte un verbe de «dire» ou de «croire» qui introduit une proposition complétive enchâssée, les déictiques qu'elle comporte s'intéprètent par rapport à l'énonciation citante. Dans notre article il n'y a qu'un cas simple, en revanche dominent les cas COMPLEXES (8): le DI comporte un segment entre guillemets (îlot textuel), qui apparaît comme repris à une énonciation de Flaubert, donc non reformulé (c'est un cas de la MA).

Une fois (9) l'attribution de la source est mise en doute. Brunetière suggère que Joseph Prud'homme, un personnage littéraire, aurait pu énoncer les paroles citées. Etant donné qu'il est une caricature du bourgeois français, le critique vise ainsi le côté banal et bourgeois de Flaubert, responsable selon lui des défauts graves des ses oeuvres; il se moque aussi de l'écrivain connu par son attitude négative envers «le bourgeois» $(15,16)$.

Le discours indirect libre (DIL) ressemble au DI quant à l'adaptation des pronoms et des temps, tandis qu'il se rapproche du DD grâce à son autonomie syntaxique, à la présence possible des exclamations, des modalisations et des déictiques spatio-temporels assumés par l'énonciateur du discours cité. Brunetière recourt à ce moyen (10) pour reprendre la caractéristique d'un personnage de Flaubert, Binet, sur laquelle il enchaîne avec une citation du roman où Binet est qualifié d' «artiste» et de «bourgeois». Il reprend ces mots, et il identifie les mêmes traits chez l'écrivain. Par conséquent, il classe les oeuvres «manquées» en celles faites par «l'artiste» (Salammbô, la Tentation de saint Antoine, Hérodias) et par «le bourgeois» (un Coeur simple, l'Education sentimentale, le Candidat, le Château des coeurs (p. 202).

Le discours narrativisé (DN): la parole est mentionnée sous forme d'un infinitif ou d'un syntagme nominal objet d'un verbe locutoire, ou encore d'un substantif locutoire abstrait (Gaulmyn 1983 dans De Arruda 1992: 25). Nous signalerons deux particularités:

1. Le critique réfère à l'écrivain par le pronom «vous», en l'instituant ponctuellement en son co-énonciateur (11). Il rapproche aussi les verbes «raconter» et «peindre» (12).

2. Les verbes tels que «esquisser» (le portrait) ou «peindre» correspondent en effet à un verbe locutoire, p.ex. «décrire».

La modalisation en discours second. Domine la MA, on rencontre les formes explicites, p.ex. «l'expression est encore de Flaubert», «ce qu'il appelle la société des femmes perdues», mais surtout ce sont les guillemets qui, compte tenu du cotexte, confèrent aux segments qu'ils entourent la valeur des mots propres de Flaubert. Le critique fait donc usage des mots sont déterminés par l'objet dont il parle. C'est le cas du discours approprié à l'objet du dire (Authier-Revuz 1995: 316). Voilà les cas les plus importants:

1. La définition du mot «artiste» (13), crucial pour juger l'écrivain, présente une combinaison de la MDS sur le contenu, marquée par la forme «comme le dit Flaubert lui-même», aux guillemets de MA. Ils marquent la reprise des principes propres de l'écrivain pour juger lui-même et son oeuvre. La façon de parler de Flaubert étant 
qualifiée comme »un peu lourde», le critique enchaîne par «c'est-à-dire», en introduisant de cette façon une manière de parler censée respecter une norme commune à lui et à ses lecteurs (Murat, Cartier-Bresson 1987: 7). Deux espaces dicursifs se rencontrent: par opposition à la manière de parler du critique, le dire de Flaubert est ici «autre» parce que l'écrivain s'exprime «un peu lourdement».

2. La caractéristique de l'écrivain reprend les mots qu'il avait utilisés lui-même, soit pour parler de son héros (14), soit pour lutter contre le «bourgeois». Plusieurs fois ce mot apparaît entre guillemets, et dans le contexte il s'intérprète comme repris à Flaubert $(15,16)$.

Le critique l'emploie aussi de façon transparente, en démontrant le côté bourgeois de l'écrivain, qui se répercute dans son oeuvre (11).

3. La reprise de petits fragments du texte de Flaubert, égaux à un syntagme ou à un énoncé (17) (p. 178, 179, 181, 182, 184, 186, etc.), intégrés syntaxiquement au discours propre du critique (qui n'est pas sans rappeler le résumé avec citations, Maingueneau 1994: 132-133). Cette manière de parler avec les mots de l'oeuvre permet de faire valoir la qualité du texte et donne aux considérations du critique un aspect objectif.

Les emprunts des segments des oeuvres sont des fragments des oeuvres qui ne sont pas supposés correspondre à des actes d'énonciation. Le plus vaste compte une page (un exemple d' «une magnifique éloquence»: la scène où on décerne une médaille à Catherine Leroux). La reprise des segments sert à:

1. Analyser des procédés que le critique apprécie. Ils sont introduits soit par le verbe «citer» (p. 155, 168, 189), soit par un syntagme nominal («procédé de peintre», p.154, «procédé», p.156, 168, «exemple» p.155, 168, «phrase», p. 155, «une comparaison», p. 156, «moyen de construire le paragraphe», p. 159, etc.);

2. Exemplifier ces aspects de la langue de Flaubert que le critique désapprouve («la raillerie», p. 187, «la surprenante impuissance de la langue» dans le domaine psychologique, p. 192);

3. Donner la formule qui selon le critique résume l'essentiel et l'oevre de Flaubert et du naturalisme (18). Il faut remarquer qu'ici il réfère à Flaubert par le syntagme «le poète».

\section{CONCLUSION}

Nous avons examiné les moyens linguistiques et discursifs par lesquels Brunetière fait place au discours de l'écrivain qui est l'objet de son analyse.

1. Pour juger l'écrivain «sur ses oeuvres», il tient compte non seulement des oeuvres, mais aussi de ses énonciations faites à diverses occasions. Cela nous permet d'envisager la notion du «discours de l'écrivain» qui embrasse tout ce qu'il a écrit ou dit.

2. Le discours de Flaubert est représenté surtout par les formes qui opaques (DD) ou opacifiantes (MA, combinées - ou non - avec le DI, la MDSc). La présence im- 
portante de la MA n'est pas surprenante dans le discours critique, qui fait partie des genres qui favorisent l'apparition du «discours approprié» (au sens de Authier-Revuz), des mots qui sont donnés par l'objet dont on parle.

3. C'est au discours de l'écrivain que le critique reprend plusieurs critères qui fondent ses jugements (la définition du mot «artiste», la distinction du côté «artiste» et «bourgeois» de Flaubert, l'appréciation des manifestations de l'aspect noble de la réalité). Il retourne contre l'écrivain ses propres mots avec le DD fictif, censé intérpréter les mots de Flaubert.

4. Les moyens utilisés par le critique pour reprendre le discours de l'écrivain permettent partiellement d'expliquer comment il exerce sa fonction de juger.

5. Le statut de co-énonciateur conféré ponctuellement à l'écrivain (11) et le fait de référer au romancier par le syntagme «le poète» (18).

\section{EXEMPLES}

(1) Et elle [Emma] a raison de dire: «Comme c'était loin!, tout cela!» Oui, comme c'était loin! mais non pas à toujours évanoui! comme c'était loin! mais comme au plus profond de sa mémoire elle en gardait le cher, et vivant, et riant souvenir! Comme c'était loin! et pourtant c'était encore près d'elle!... (p. 160).

(2) «Combien d'écrivains parmi les plus vantés, dit-il lui-même en parlant de Louis Bouilhet, seraient incapables de faire une narration, de joindre bout à bout une analyse, un portrait, un dialogue?» Il élevait Bouilhet trop haut... mais le mérite qu'il signale, il avait raison de le vanter; ... il avait raison encore, s'il se rendait intérieurement le témoignage, lui, Flaubert, de l'avoir eu (p. 167).

(3) «Son spiritualisme, dit-il d'une de ses héroünes, - madame Dambreuse croyait à la transmigration des âmes - ne l'empêchait pas de tenir sa caisse admirablement». Et pourquoi, bon Dieu! son spiritualisme l'aurait-il empêchée de «tenir admirablement sa caisse? (191).

(4) C'est à peu près comme s'il avait dit: Quoi de plus antihumain qu'une amitié qui ne dégénère pas en compagnonnage? et quoi de plus «spécial» que la dignité de la tenue? Il y a certainement une lacune dans sa connaissance de l'homme (192).

(5) «Les uns voient bleu, dit-il quelque part, les autres voient noir; la multitude voit bête». C'est sa devise. Je n'ai pas besoin d'en faire linguement ressortir la fausseté. La multitude ne voit pas «bễte», elle voit «banal» ... (197).

(6) ... en travaillant ... à se perfectionner dans le mépris de l'homme, en même temps que dans le maniement de son matériel, il a oublié que l'ironie était fatalement inféconde. «La désillusion est le propre des faibles. Méfiez-vous des dégoûtés, ce sont presque toujours des impuissants». C'est lui-même qui l'a dit, et très bien dit. (199).

(7) ... il se dissimule souvent, et des idées saines, et des sentiments vrais, et des intentions délicates sous les apprences de la sottise et de la naveté. Il le savait sans 
doute, puisqu'il a dit encore lui-même: «Comme si la plénitude de son âme ne débordait pas par les métaphores les plus vides». Oui! par les métaphores les plus vides; et par les gestes les plus étranges; et par les actes les plus imprévus! (199).

(8) N'at-il pas écrit encore... en comparant désobligeamment l'eunuque Schahabarim aux «bonshommes de Port Royal», qu'après tout «Schahabarim lui semblait moins antihumain, moins spécial, moins cocasse que des gens vivant en commun et qui s’appellent jusqu'à la mort: Monsieur?» (192).

(9) Mais lorsque parlant toujours en son nom personnel il nous apprend que «le sieur Arnoux se livrait à des espiègleries côtoyant la turpitude», ô Muse du naturalisme! est-ce Flaubert qui parle, ou si c'est M. Prud'homme? (198).

(10) Là bas ... Binet ... tourne avec rage. Il y en a qui aiment autour de lui; il y en a qui meurent. Que lui importe! et qu'a-t-il lui, Binet, avec tous ces gens là? Leurs affaires ne sont pas les siennes! [je souligne] Et tourant ... avec rage, il fabrique «des ronds de serviette, dont il encombre sa maison avec la jalousie d'un artiste et l'égoïsme d'un bourgeois». Il y eut de cet artiste et de ce bourgeois dans Flaubert (202).

(11) Vous vous moquiez du bourgeois! [soulignement A.D.] Le bourgeois vous l'a rendu cruellement le jour qu'il vous inspira l'Education sentimentale (201).

(12) Quand ... Flaubert nous raconte les terreurs de Carthage ... et qu'il nous peint le bout de tableau que voici... [soulignement A.D.].

(13) Si, comme le dit Flaubert lui-même, - un peu lourdement, - ... si «les accidents du monde, dès qu'ils sont perçus, vous apparaissent comme transposés pour l'emploi d'une illusion à décrire, tellement que toutes les choses, y compris votre existence, ne vous semblent pas avoir d'autre utilité», c'est-à-dire, si vous considérez le monde, la nature, la vie, l'homme enfin comme des choses qui seraient faites pour l'art, et non plus l'art comme une chose qui serait faite pour l'homme, vous êtes artiste, au sens entier du mot, dans la force et dans la profondeur du terme (150).

(14) Flaubert ne laissa pas de ressembler à son curé Bournisien: il avait comme lui, «la stature athlétique»; il a souvent, comme lui, «le rire opaque» (199).

(15) ... un peu de son mépris d'artiste pour le «bourgeois»... (178).

(16) ... cette même haine inepiable du «bourgeois»... (202).

(17) Moins habituée aux «aspects calmes», elle [Emma Bovary] ne se serait par tournée vers les «accidentés» (178).

(18) C'est le point culminant du drame. Voici de quels traits le poète l'a marqué: «Jamais Madame Bovary ne fut plus belle qu'à cette époque; elle avait cette indéfinissable beauté qui résulte de la joie, de l'enthousiasme, du succès et qui n'est que l'harmonie du tempérament avec les circonstances. Ses convoitises, ses chagrins, l'expérience du plaisir et ses illusions toujours jeunes, comme font aux fleurs le fumier, la pluie, les vents et le soleil, l'avaient, par gradations, développée, et elle s'épanouissait enfin dans la plénitude de sa nature». Pesez bien ces deux phrases: elles sont tout le roman, tout Flaubert, tout le système, toute l'école, tout le naturalisme (182). 


\section{BIBLIOGRAPHIE}

Authier-Revuz, J. (1982), Hétérogénéité montrée et hétérogénéité constitutive: éléments pour une approche de l'autre dans le discours, DRLAV 26: 91-151.

A u thi er-Revuz, J. (1992), Repères dans le champ du discours rapporté, L'Information grammaticale 55: 38-42.

A u thier-Revuz, J. (1993), Repères dans le champ du discours rapporté (suite), L'Information grammaticale $56: 10-15$.

Authier-Revuz, J. (1995), Ces mots qui ne vont pas de soi. Boucles réflexives et non-coincidences $d u$ dire, t. 1-2, Larousse: Paris.

A u thi er-Revuz, J. (1997), Modalisation autonymique et discours autre, Modèles linguistiques, t. XVIII, fasc. 1: 33-51.

Brunetière, F. (1880), Le naturalisme français. Etude sur Gustave Flaubert, dans: Le roman naturaliste, s.d., Calmann-Lévy: Paris.

De Arruda, D. (1992), Discours rapporté et circulation de la parole. Contribution à une approche dialogique du discours d'autrui (Etude de six commentaires oraux induits par la lecture d'un article de presse), Peeters, Louvain-La-Neuve.

Ga ul m y n M.-M. de (1983), Les verbes de communication dans la structuration du discours: essai sur la réfléxivité du langage - Récits d'enfants et échanges entre enfants et adultes. Thèse d'Etat, Université de Lyon et Université Paris VIII.

Jaubert A., Labirynthes énonciatifs, Modèles linguistiques, t. XVIII, fasc. 1: 17-31.

Kroker W., Sobies ka-Pił a to wicz A. (1997), Anthologie de la critique littéraire française des $X I X^{e}$ et $X X^{e}$ siècles. Uniwersytet Warszawski, Instytut Romanistyki: Warszawa.

Mainguen eau D. (1994), L'énonciation en linguistique française, Hachette, Paris.

Mo i rand S. (1988), Une histoire de discours... Une analyse de discours de la revue "Le Français dans le monde» 1961-1981, Hachette: Paris.

Mortara-Garavelli B. (1985), La parola d'altri. Prospettive di analisi di discorso. Sellerio editore, Palermo.

Mortara-Garavelli B. (1995), Il discorso indiretto nell'italiano parlato, Etudes Romanes 34: 69-88.

Murat M., Cartier-Bresson B. (1987), C'est-à-dire ou la reprise intérprétative, Langue française 73: 5-15.

Perrin L. (1994), Mots et énoncés mentionnés dans le discours, Cahiers de linguistique française 15: $217-248$.

Rosier L. (1997), Entre binarité et continuum, une nouvelle approche théorique du discours rapporté? Modèles linguistiques t. XVIII, fasc. 1: 7-16.

Si mon in J. (1984), Les plans d'énonciation dans Berlin Alexanderplatz de Döblin, ou de la polyphonie textuelle, Langages 73: 30-56. 